\title{
Quercetin Affects Spermatogenesis-Related Genes of Mouse Exposed to High-Cholesterol Diet
}

\author{
Changwon Yang ${ }^{1}$, Hyocheol Bae ${ }^{1}$, Gwonhwa Song ${ }^{1, *}$ and Whasun Lim ${ }^{2, *}$ \\ ${ }^{1}$ Institute of Animal Molecular Biotechnology and Department of Biotechnology, College of Life Sciences and \\ Biotechnology, Korea University, Seoul 02841, Korea \\ ${ }^{2}$ Department of Food and Nutrition, College of Science and Technology, Kookmin University, Seoul 02707, Korea
}

Received March 3, 2020

Revised March 10, 2020

Accepted March 10, 2020

*Correspondence

Gwonhwa Song

E-mail: ghsong@korea.ac.kr

ORCID

https://orcid.org/0000-0003-2817-5323

Whasun Lim

E-mail: wlim@kookmin.ac.kr

ORCID

https://orcid.org/0000-0002-1328-0465

\begin{abstract}
A high-cholesterol diet can reduce male fertility. However, it is not known whether a high-cholesterol diet can regulate the expression of genes involved in sperm maturation and sperm fertilizing ability. Quercetin, a natural product, is known to have cytoprotective effects by regulating lipid metabolism in various cell types. This study aimed to confirm the expression of genes involved in sperm maturation in the testes of mice fed a high-cholesterol diet and to determine whether quercetin can reverse the genetic regulation of cholesterol. Mice were divided into groups fed a normal chow diet and a high-cholesterol diet. Mice fed the high-cholesterol diet were dose-dependently supplemented with quercetin for 6 weeks. Investigations using quantitative PCR and in situ hybridization revealed that the high-cholesterol diet alters the expression of genes associated with sperm maturation in the testes of mice, and this was reversed with the supplementation of quercetin. In addition, the high-cholesterol diet regulated the expression of genes related to lipid metabolism in the liver of mice. Under a highcholesterol diet, quercetin can improve male fertility by regulating the expression of genes involved in sperm maturation.
\end{abstract}

Keywords: cholesterol, lipid metabolism, quercetin, spermatogenesis, testis

\section{INTRODUCTION}

Approximately $30 \%$ of cases of male infertility have no known cause (Cavallini, 2006). Several studies have shown that obesity-related diseases are associated with a decrease in male reproductive potential, which is evidenced by a decrease in sperm parameters and testosterone levels (Lotti et al., 2013; Leisegang et al., 2014). The testis is the organ responsible for fertility by producing sperm and it functions through the physiological action of various types of cells, including Leydig cells, Sertoli cells, germ cells, and peritubular cells (Majumdar and Bhattacharya, 2013). Cholesterol is a structural component that maintains the fluidity of cell membranes and plays an important role in regulating the physiological function of mammalian cells. Moreover, cholesterol is a precursor to steroid synthesis, which is essential for the production of normal sperm (Yokoyama, 2000). Cholesterol homeostasis is also disturbed by exposure to environmental toxins, which in turn disrupts spermatogenesis and the development of gonads (Samova et al., 2018). In rabbits, hypercholesterolemia damages Sertoli and Leydig cells in the testes and reduces sperm quality (Saez Lancellotti et al., 2010). Moreover, in male mice fed a high-cholesterol diet, the testes have been shown to have low steroidogenic enzymes and high oxidative stress (Wang et al., 2015). How- 
ever, it is not known whether a cholesterol diet can affect the expression of genes related to sperm maturation and sperm fertilizing ability.

Quercetin (3,3', 4',5,7-pentahydroxyflavone) is a polyphenolic flavonoid widely found in vegetables and fruits and has been shown to improve various health functions with anti-cancer, antioxidant, and anti-inflammatory effects. Quercetin also alters gene transcription and can affect signal transduction pathways that regulate cell proliferation (Brito et al., 2015). In addition, quercetin has been shown to reduce cholesterol synthesis and regulates lipid metabolism in several cell types, including pancreatic $\beta$-cells and glial cells (Carrasco-Pozo et al., 2016; Damiano et al., 2019). Moreover, quercetin is known to mitigate the harmful effects of various chemicals that damage the testes and cause reproductive toxicity in mammals (Izawa et al., 2008; Abdel Aziz et al., 2018). However, it is unclear whether quercetin can control genetic changes in the testes caused by a high-cholesterol diet.

In our previous studies, we found that high-cholesterol diets cause histological alterations in the male reproductive organs in rodents (Lim et al., 2015). In this study, we examined the change in sperm adhesion molecule 1 (Spam1), ADAM metallopeptidase domain 5 (Adam5), Adam29, steroid 5 alpha-reductase 2 (Srd5a2), and Srd5a3 that play a role in sperm maturation and sperm fertilizing ability in the testes of mice fed a high-cholesterol diet. In addition, quercetin was added to the feed to determine whether the changes in gene expression caused by cholesterol could be controlled. Moreover, we investigated the expression of lipid metabolism-related genes in the liver tissues of mice fed high-cholesterol and quercetin supplements. Taken together, we verified that quercetin could reverse changes caused by high-cholesterol diets in the expression of genes responsible for sperm quality in the testis.

\section{MATERIALS AND METHODS}

\section{Experimental animals and diets}

The experimental use of mice in this study was approved by the Animal Care and Use Committee of Korea University. Four-week-old male C57BL/6 mice were maintained for an adaptive period of 10 days with ad libitum diet in conditions of $12 \mathrm{~h}$ light and $12 \mathrm{~h}$ dark, according to the institutional guidelines for animal care. Afterward, a total of 48 mice were divided into six groups. Group 1 $(\mathrm{n}=8)$ was fed a normal diet and Groups 2 through 6 ( $\mathrm{n}=$ 8 per each group) were fed a high-cholesterol diet containing 3\% cholesterol and $0.5 \%$ cholic acid. Groups 3, 4, and 5 were additionally fed 25,50 , and $100 \mathrm{mg} / \mathrm{kg}$ quercetin, respectively. Finasteride $(10 \mathrm{mg} / \mathrm{kg})$ was added to the feed of group 6 . Each diet was provided for 6 weeks. The composition of normal and high-cholesterol diets is provided in Table 1.

\section{Tissue samples}

Mice were euthanized after starving for $12 \mathrm{~h}$. The testis and liver tissues were then stored in liquid nitrogen for RNA extraction and in $4 \%$ paraformaldehyde in phosphate buffered saline for tissue fixation.

\section{Quantitative RT-PCR analysis}

Trizol (Invitrogen, Carlsbad, CA, USA) was used to extract the total RNA. AccuPower RT premix (Bioneer, Daejeon, Korea) was used to synthesize complementary DNA. Gene expression was measured using a StepOnePlus RealTime PCR System (Applied Biosystems, Foster City, CA, USA) using SYBR Green (Sigma, St. Louis, MO, USA). The $\mathrm{Ct}$ value, i.e., the number of cycles in which the fluorescence signal is statistically greater than that of the background, was used to quantify the expression of the target gene based on the $2^{-\Delta \Delta C T}$ method. The primer sets used are listed in Table 2. Expression of Gapdh was used to normalize the expression of target genes.

Table 1. Percentage composition of normal chow and the highcholesterol diet

\begin{tabular}{lcc}
\hline \multicolumn{1}{c}{ Ingredients } & Chow diet & High-cholesterol diet \\
\hline Casein & 20 & 20 \\
Corn starch & 40 & 36.5 \\
Maltodextrin & 13.2 & 13.2 \\
Sucrose & 7 & 7 \\
Tallow & 8 & 8 \\
Soybean oil & 2 & 2 \\
Cellulose & 5 & 5 \\
Mineral mixture & 3.5 & 3.5 \\
Vitamin mixture & 1 & 1 \\
DL-methionine & 0.3 & 0.3 \\
Cholic acid & - & 0.5 \\
Cholesterol & - & 3 \\
\hline
\end{tabular}


Table 2. Primer sets used in quantitative RT-PCR analyses

\begin{tabular}{lll}
\hline $\begin{array}{c}\text { Gene } \\
\text { symbol }\end{array}$ & \multicolumn{1}{c}{$\begin{array}{c}\text { Sense primer } \\
\left(\mathbf{5}^{\prime} \rightarrow \mathbf{3}^{\prime} \mathbf{)}\right.\end{array}$} & \multicolumn{1}{c}{$\begin{array}{c}\text { Antisense primer } \\
\left(\mathbf{5}^{\prime} \boldsymbol{\prime} \rightarrow \mathbf{3}^{\prime} \mathbf{)}\right.\end{array}$} \\
\hline $\begin{array}{l}\text { Gapdh } \\
\text { Spam1 }\end{array}$ & AACTTTGGCATTGTGGAAGG & ATGCAGGGATGATGTTCTGG \\
Adam5 & AGGAGAATCTGTGGCAATGG & CCGTGCAATCTTGACTACAGC \\
Adam29 & CCATGAATGTCCAGATGATGC & TTGCCTACAGTGCTCATTGC \\
Srd5a2 & AGAGGCAGGCCTTTATCAGC & GAAGACACCGACGCTAAACC \\
Srd5a3 & CCGCCCATCAGTATAAATGC & CTCGAACCAGTCTCCAAAGG \\
Mxlipl & CTTCAAAGGCCTCAAGTTGC & GTCACGAAACCACACACTGG \\
Ppara & GCAGATGACCTGGAAGTCC & TCTGCAAAACCAAAGCTTCC \\
Cd36 & AAAATCGTGGAGTGGAATGG & CTGACGGGAAGAGGTACAGG \\
Fabp1 & GAGCCAGGAGAACTTTAGC & CATGCACGATTTCTGACACC \\
Fatp5 & TCGGATCTGGGAATTCTACG & CAAGCTCAAAGGGAGTCAGC \\
Cpt1 & GACAGACACCATCCAACACG & AGGTGTCATCCAGGATCTGC \\
\hline
\end{tabular}

\section{In situ hybridization analysis}

PCR products generated from cDNA were cloned using TOPO vector (Invitrogen) to synthesize hybridization probes. Plasmids with the correct gene sequence verified were amplified using T7- and SP6-specific primers. The DIG RNA labeling kit (Roche, Indianapolis, IN, USA) was then used to transcribe DIG-labeled RNA probes. Testis tissues fixed in $4 \%$ paraformaldehyde were embedded in paraffin blocks and sectioned ( $5 \mu \mathrm{m}$ thick) on slide glass. The sections were deparaffinized with xylene and rehydrated in DEPC-treated water containing a gradual alcohol series. Then, the sections were treated with $1 \%$ Triton X-100 for 20 min and washed twice with DEPC-treated phosphate buffered saline. The sections were then digested at $37^{\circ} \mathrm{C}$ with $5 \mu \mathrm{g} / \mathrm{mL}$ of proteinase $\mathrm{K}$ (Sigma) diluted in TE buffer. Next, the sections were exposed twice for 5 min in a buffer containing $0.25 \%(\mathrm{v} / \mathrm{v})$ acetic anhydride and $0.1 \mathrm{M}$ triethanolamine. The sections were exposed for $10 \mathrm{~min}$ at room temperature in a prehybridization mixture containing $50 \%$ formamide and $4 \mathrm{X}$ standard saline citrate (SSC). They were then incubated in a hybridization mixture and DIG-labeled cRNA probe for $16 \mathrm{~h}$ at $42^{\circ} \mathrm{C}$. The sections were washed for $15 \mathrm{~min}$ in $2 \mathrm{X}$ SSC, 30 min in $1 \mathrm{X}$ SSC, 30 min in NTE buffer, and 30 min in 0.1X SSC. Normal sheep serum (2\%) was used for blocking and sections were exposed for $16 \mathrm{~h}$ to sheep anti-DIG antibody conjugated with alkaline phosphatase. Signals for visualizing the expression of target genes were detected using solutions containing 5-bromo-4-chloro-3-indolyl phosphate, nitroblue tetrazolium, and levamisole.

\section{Statistical analyses}

The statistical significance of quantitative PCR cells was confirmed based on the analysis of variance using SAS (SAS Institute). Differences with a probability value of $p<0.05$ were considered statistically significant. Data are presented as mean \pm SEM unless otherwise stated.

\section{RESULTS}

\section{Effect of quercetin supplementation on high-cholesterol diet-altered expression of Spam 1}

We first confirmed the mRNA level of the Spam1 gene in the testes of mice fed the high-cholesterol diet using quantitative PCR (Fig. 1A). Spam1 expression was reduced by $56.0 \%(p<0.001)$ in the testes of mice fed a high-cholesterol diet compared to Spam1 expression in mice fed a normal chow diet. We added quercetin to the feed in a dose-dependent manner $(0,25,50$, and $100 \mathrm{mg} / \mathrm{kg})$ to determine if the effect of reduced expression of Spam1 owing to the high-cholesterol diet could be reversed by quercetin. We confirmed that $25 \mathrm{mg} / \mathrm{kg}$ of quercetin restored the mRNA level of Spam1 to that of the control and found that even higher doses of quercetin increased the expression of SPAM1 in a dose-dependent manner. Finasteride, a competitive inhibitor of $5 \alpha$-reductase, responsible for the conversion of testosterone to DHT, the most active androgen, restored the expression of Spam1, reduced by a high-cholesterol diet, similar to that by quercetin. Using in situ hybridization assays, we histologically identified that quercetin can restore the expression of the Spam1 gene reduced owing to a high-cholesterol diet (Fig. 1B). In the testes of mice supplemented with high-cholesterol and $100 \mathrm{mg} / \mathrm{kg}$ of quercetin, Spam 1 was highly expressed in the spermatogonia (Spg), spermatocytes (Spc), spermatids (Spt), and spermatozoa (Spz). Spg showed the highest expression of Spam1 during sperm maturation in the group fed quercetin supplementation. Spam1, in contrast, was weakly detected in the testes from mice fed a highcholesterol diet without quercetin supplementation.

\section{Effect of quercetin supplementation on high-cholesterol} diet-altered expression of Adam5 and Adam29

Next, we identified the effects of high-cholesterol diets and quercetin supplementation on the expression of the Adam 5 and Adam 29 genes. The high-cholesterol diet increased the mRNA levels of the Adam5 gene 1.5-fold 


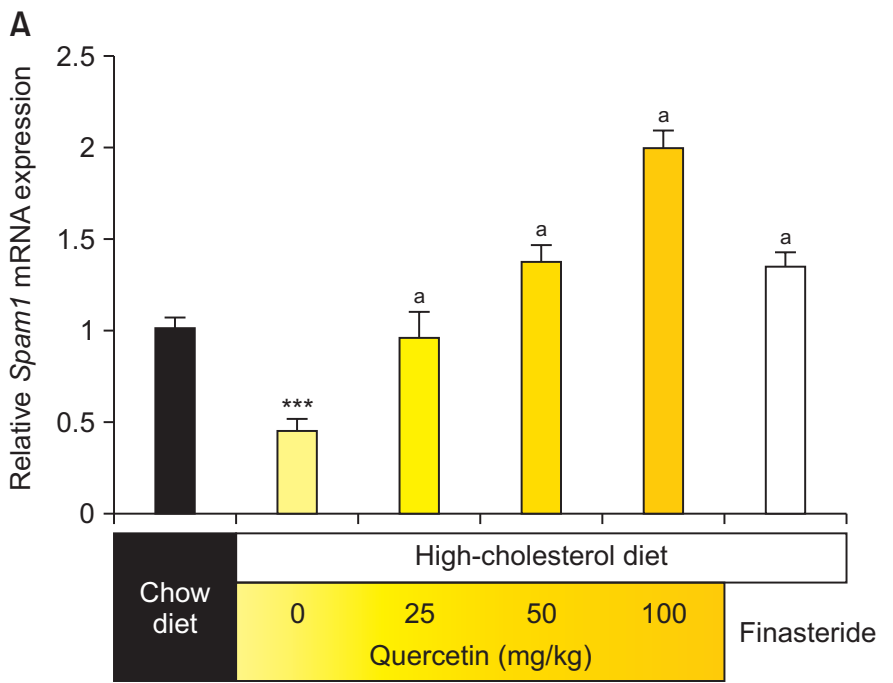

B
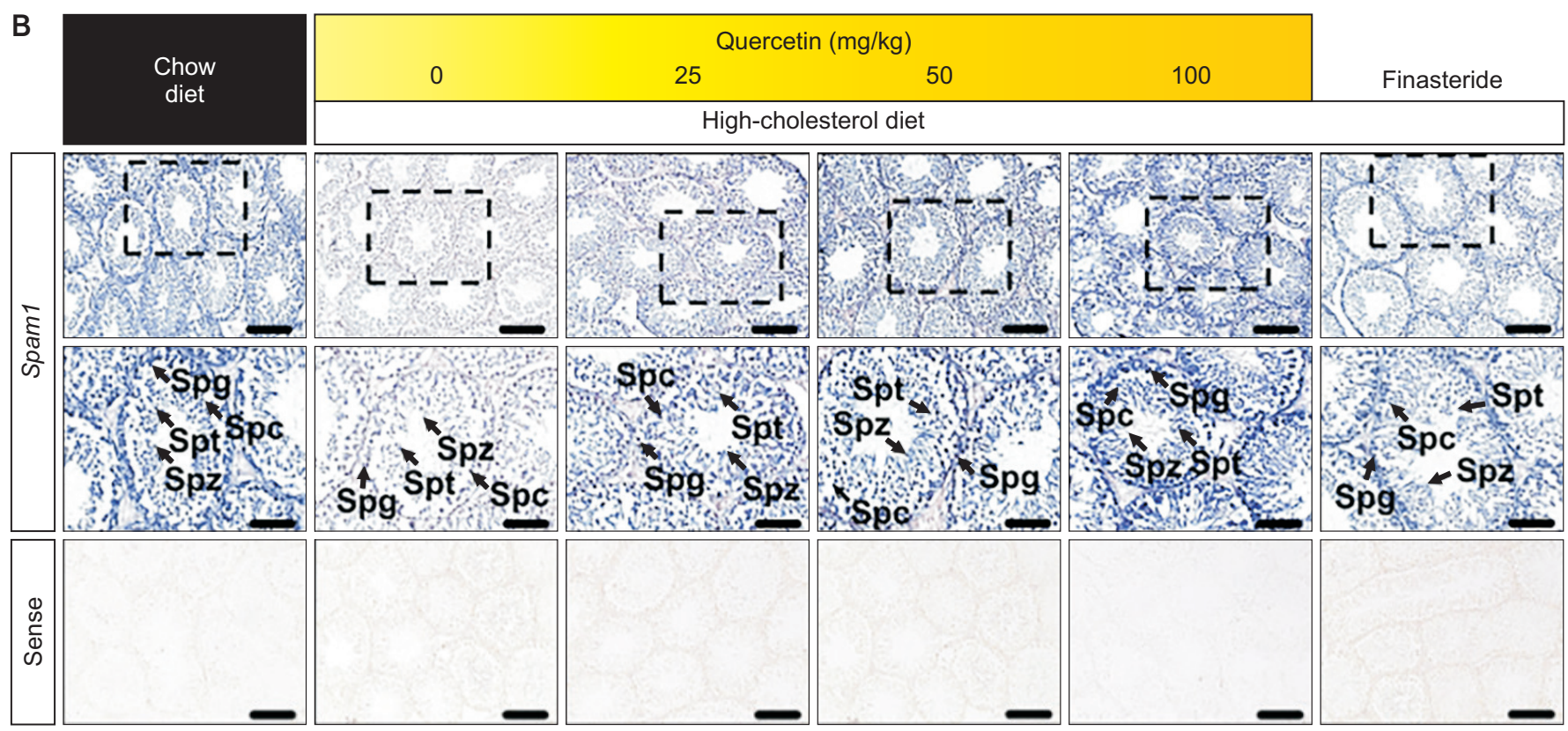

Fig. 1. Effects of a high-cholesterol diet and quercetin supplementation on the expression of Spam1 in the testes of male mice. (A) Using quantitative RT-PCR, Spam 1 mRNA levels were estimated in the testes of mice fed normal chow and a high-cholesterol diet. Quercetin $(0,25,50$, and $100 \mathrm{mg} / \mathrm{kg})$ and finasteride $(10 \mathrm{mg} / \mathrm{kg})$ were added to a high-cholesterol diet and Spam $1 \mathrm{mRNA}$ levels were investigated in the testes. The relative quantities of mRNA were normalized to the quantity of Gapdh mRNA. The asterisks denote significant differences $\left({ }^{* * *} p<0.001\right)$. The letter "a" means that there is a significant difference in the expression of Spam 1 in the group with quercetin or finasteride supplementation as compared to the expression of Spam 1 in the high-cholesterol diet group. (B) Localization of Spam 1 mRNA in the testes was detected through in situ hybridization analyses. Cross and longitudinal sections of the testes were hybridized with antisense or sense Spam1 cRNA probes. Scale bars represent $10 \mu \mathrm{m}$ (the first horizontal panels and sense) and $5 \mu \mathrm{m}$ (the second horizontal panel). Spc, spermatocyte; Spg, spermatogonium; Spt, spermatid; Spz, spermatozoa.

$(p<0.01)$ in the testes of male mice (Fig. 2A). Meanwhile, feeding quercetin or finasteride reduced Adam5 expression to levels similar to that in the testes of mice fed normal chow diet. Histologic analysis of the testes also showed increased expression of Adam5 by high-cholesterol diet compared to normal chow diet but decreased with the addition of quercetin (Fig. 2B). The expression of
Adam5 in Spg, Spc, Spt, and Spz in the testes of mice fed a high-cholesterol diet was stronger than in mice fed a normal chow diet. Spg and Spc showed the highest levels of Adam 5 expression in the testes of mice fed a high-cholesterol diet. In contrast, weak expression of Adam5 was observed in Spg, Spc, Spt, and Spz in the testes of mice fed diets supplemented with quercetin or finasteride. 
章

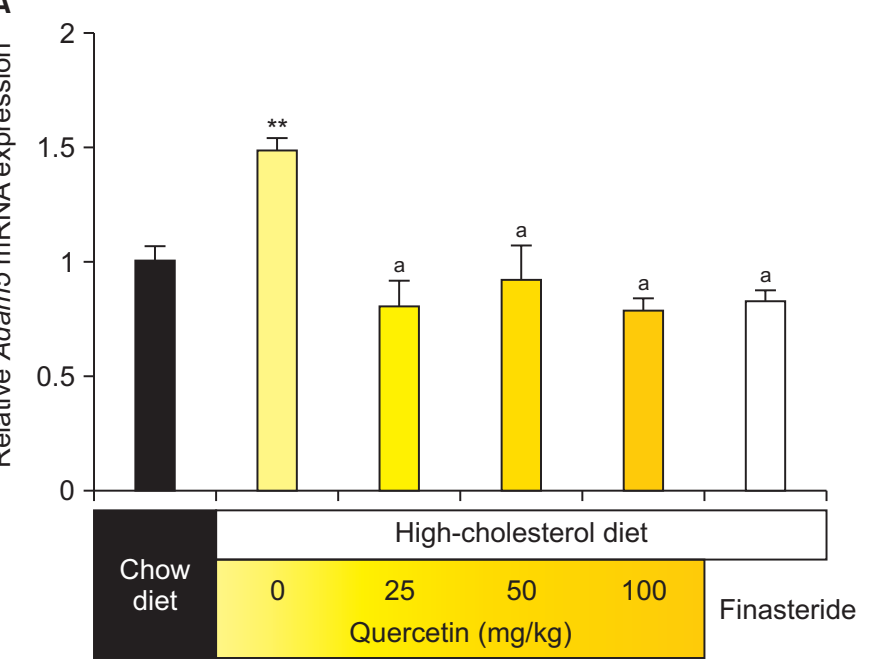

B
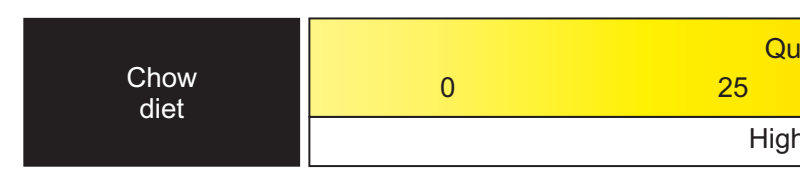

. 


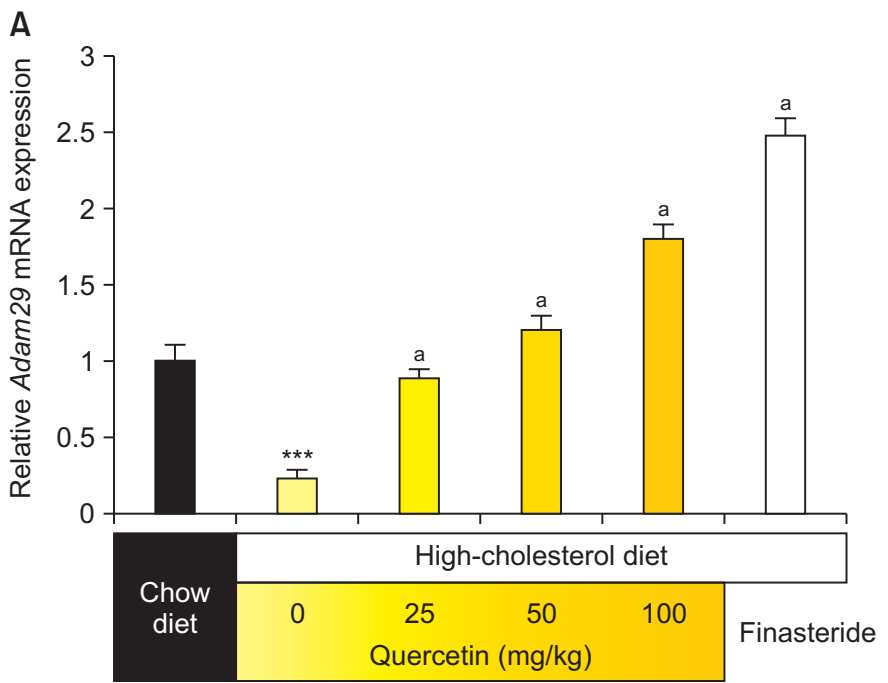

B

\begin{tabular}{|c|c|c|c|c|c|}
\hline \multirow{3}{*}{$\begin{array}{c}\text { Chow } \\
\text { diet }\end{array}$} & \multicolumn{4}{|c|}{ Quercetin (mg/kg) } & \multirow[b]{2}{*}{ Finasteride } \\
\hline & 0 & 25 & 50 & 100 & \\
\hline & \multicolumn{4}{|c|}{ High-cholesterol diet } & \\
\hline
\end{tabular}
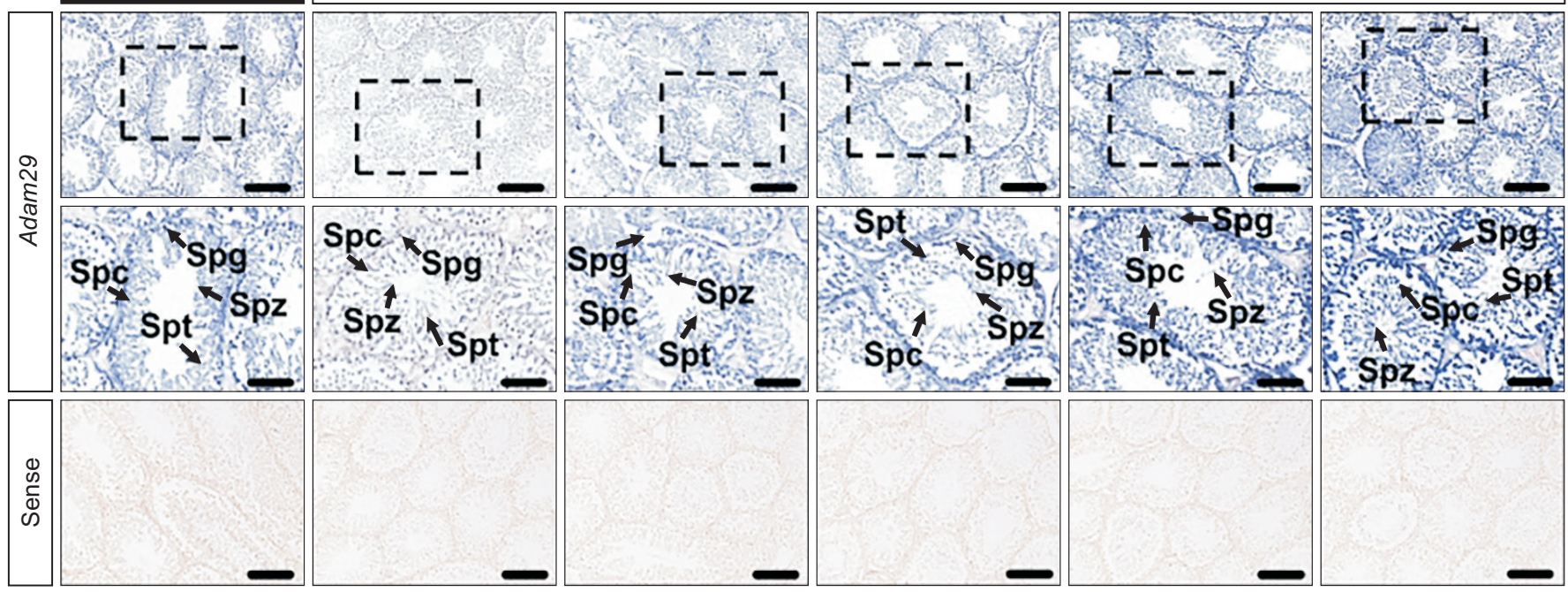

Fig. 3. Effects of a high-cholesterol diet and quercetin supplementation on the expression of Adam29 in the testes of male mice. (A) Using quantitative RT-PCR, Adam29 mRNA levels were estimated in the testes of mice fed normal chow and a high-cholesterol diet. Quercetin $(0,25,50$, and $100 \mathrm{mg} / \mathrm{kg})$ and finasteride $(10 \mathrm{mg} / \mathrm{kg})$ were added to a high-cholesterol diet and Adam29 mRNA levels were investigated in the testes. The relative quantities of mRNA were normalized to the quantity of Gapdh mRNA. The asterisks denote significant differences $\left({ }^{* *} p<0.001\right)$. The letter 'a' means that there is a significant difference in the expression of Adam29 in the group with quercetin or finasteride supplementation as compared to that in the high-cholesterol diet group. (B) Localization of Adam 29 mRNA in the testes was detected through in situ hybridization analyses. Cross and longitudinal sections of the testes were hybridized with antisense or sense Adam 29 cRNA probes. Scale bars represent $10 \mu \mathrm{m}$ (the first horizontal panels and sense) and $5 \mu \mathrm{m}$ (the second horizontal panel). Spc, spermatocyte; Spg, spermatogonium; Spt, spermatid; Spz, spermatozoa.

of mice with a high-cholesterol diet, Adam29 was rarely expressed in Spg, Spc, Spt, and Spz. However, with the addition of quercetin, high levels of Adam29 were observed during sperm maturation, especially in Spg.

\section{Effect of quercetin supplementation on high-cholesterol diet-altered expression of Srd5a2 and Srd5a3}

In the testes of mice with a high-cholesterol diet, $\operatorname{Srd} 5 \mathrm{a} 2$ expression increased 6.8-fold ( $p<0.001$ ) (Fig. 4A). The addition of 25,50 , and $100 \mathrm{mg} / \mathrm{kg}$ of quercetin in a highcholesterol diet reduced Srd5a2 expression by $57.7 \%$, $65.0 \%$, and $80.9 \%$, respectively, compared to the $\operatorname{Srd} 5 \mathrm{a} 2$ 


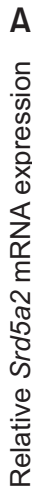

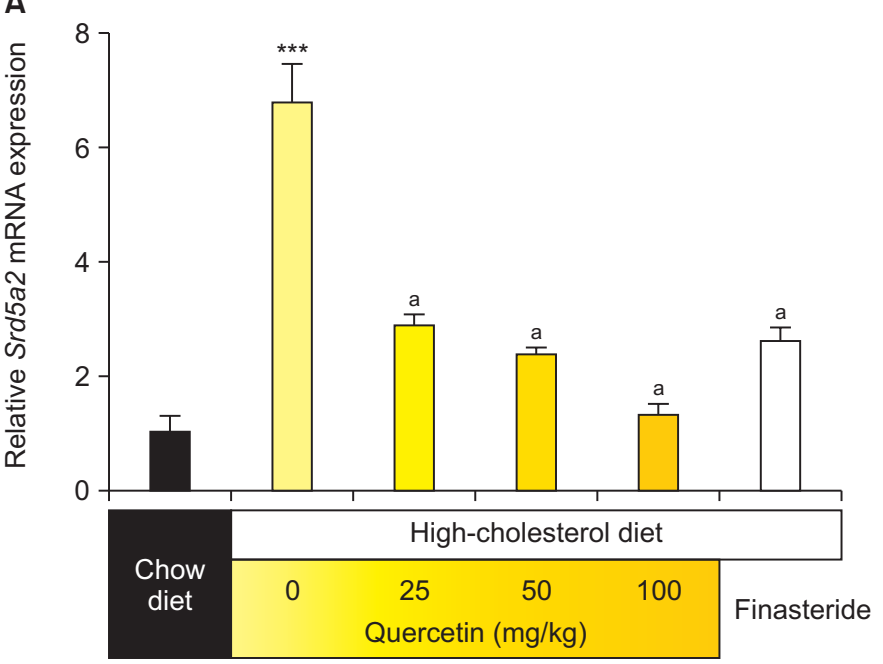

B
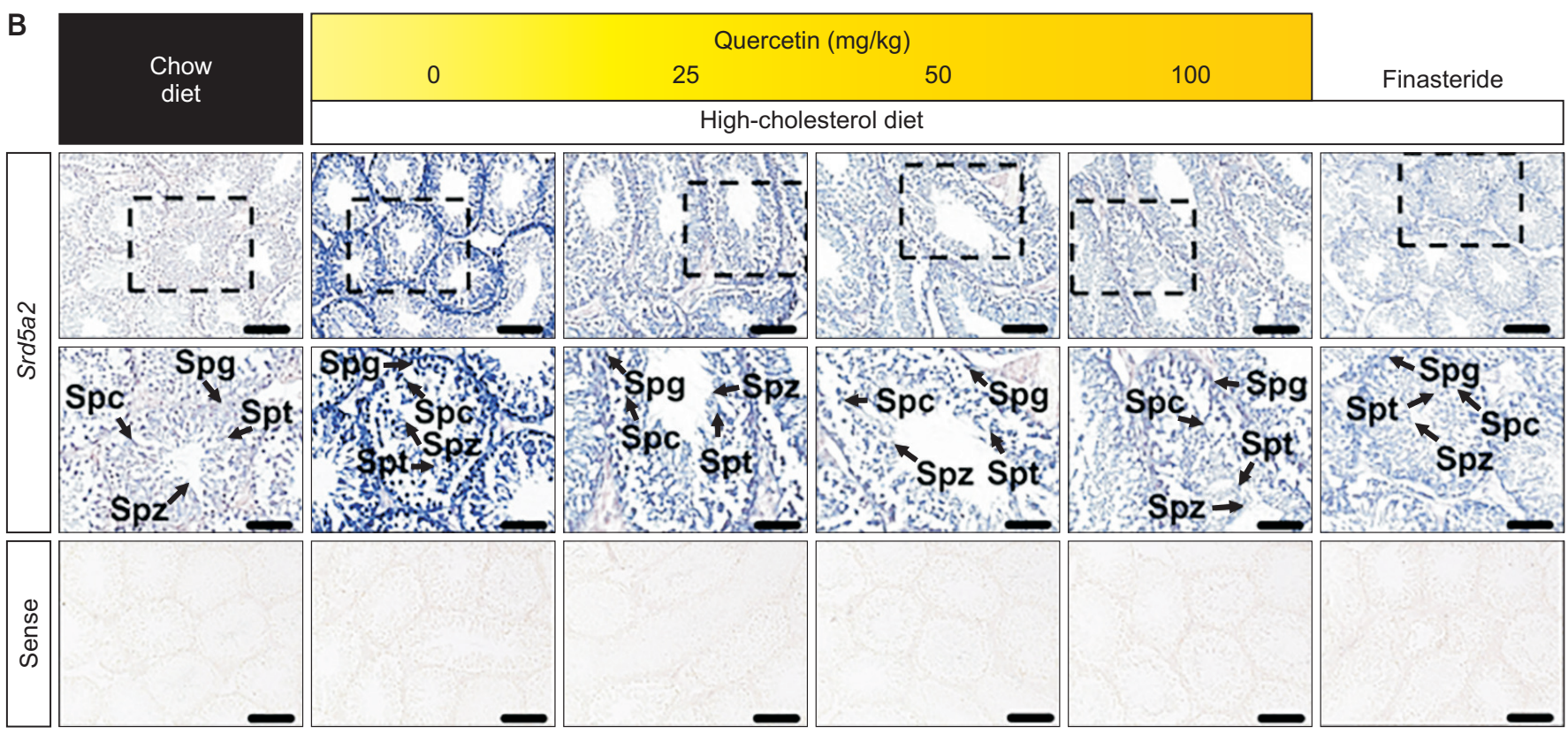

Fig. 4. Effects of a high-cholesterol diet and quercetin supplementation on the expression of Srd5a2 in the testes of male mice. (A) Using quantitative RT-PCR, Srd5a2 mRNA levels were estimated in the testes of mice fed normal chow and a high-cholesterol diet. Quercetin $(0,25,50$, and $100 \mathrm{mg} / \mathrm{kg})$ and finasteride $(10 \mathrm{mg} / \mathrm{kg})$ were added to a high-cholesterol diet and Srd5a2 mRNA levels were investigated in the testes. The relative quantities of mRNA were normalized to the quantity of Gapdh mRNA. The asterisks denote significant differences $\left({ }^{* * *} p<0.001\right)$. The letter 'a' means that there is a significant difference in the expression of Srd5a2 in the group with quercetin or finasteride supplementation as compared to that in the high-cholesterol diet group. (B) Localization of Srd5a2 mRNA in the testes was detected through in situ hybridization analyses. Cross and longitudinal sections of the testes were hybridized with antisense or sense Srd5a2 cRNA probes. Scale bars represent $10 \mu \mathrm{m}$ (the first horizontal panels and sense) and $5 \mu \mathrm{m}$ (the second horizontal panel). Spc, spermatocyte; Spg, spermatogonium; Spt, spermatid; Spz, spermatozoa.

expression for the high-cholesterol diet. The addition of finasteride also reduced $\mathrm{Srd} 5 \mathrm{a} 2$ expression by approximately $61.9 \%$ compared to that for the high-cholesterol diet. In situ hybridization analysis also revealed that the increased expression of $\mathrm{Srd} 5 \mathrm{a} 2$ in the testes of mice owing to the high-cholesterol diet decreased with quercetin addition (Fig. 4B). Srd5a2 was strongly expressed in Spg,
Spc, Spt, and Spz of mice fed the high-cholesterol diet.

Similar to Srd5a2, the expression of Srd5a3 in the testes was also increased 1.7-fold (Fig. 5A) owing to the highcholesterol diet. In addition, quercetin supplementation lowered the expression of Srd5a3 that was increased by the high-cholesterol diet compared with the expression of Srd5a3 in the testes of mice fed a normal chow diet. His- 


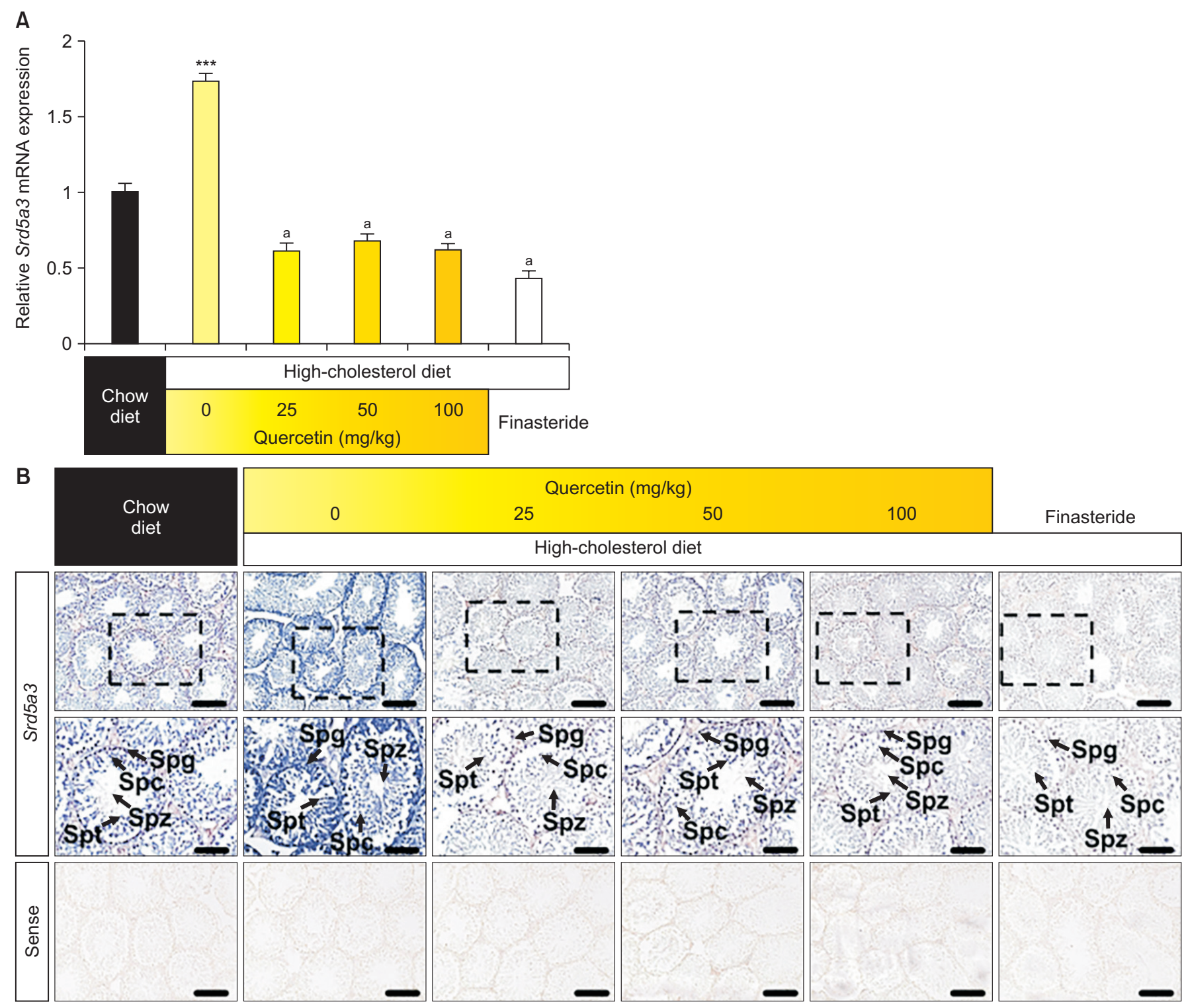

Fig. 5. Effects of a high-cholesterol diet and quercetin supplementation on the expression of Srd5a3 in the testes of male mice. (A) Using quantitative RT-PCR, Srd5a3 mRNA levels were estimated in the testes of mice fed normal chow and a high-cholesterol diet. Quercetin $(0,25,50$, and $100 \mathrm{mg} / \mathrm{kg})$ and finasteride $(10 \mathrm{mg} / \mathrm{kg})$ were added to a high-cholesterol diet and Srd5a3 mRNA levels were investigated in the testes. The relative quantities of mRNA were normalized to the quantity of Gapdh mRNA. The asterisks denote significant differences $\left({ }^{* *} p<0.001\right)$. The letter 'a' means that there is a significant difference in the expression of Srd5a3 in the group with quercetin or finasteride supplementation as compared to that in the high-cholesterol diet group. (B) Localization of Srd5a3 mRNA in the testes was detected through in situ hybridization analyses. Cross and longitudinal sections of the testes were hybridized with antisense or sense Srd5a3 cRNA probes. Scale bars represent $10 \mu \mathrm{m}$ (the first horizontal panels and sense) and $5 \mu \mathrm{m}$ (the second horizontal panel). Spc, spermatocyte; Spg, spermatogonium; Spt, spermatid; Spz, spermatozoa.

tological analysis also revealed that quercetin may lower the increased expression of $\mathrm{Srd} 5 \mathrm{a} 3 \mathrm{3}$ in the testes owing to a high-cholesterol diet (Fig. 5B). Srd5a3 expression was especially high in Spg, Spt, and Spc owing to the highcholesterol diet.
Expression of genes involved in lipid metabolism with the high-cholesterol diet and quercetin supplementation

Next, we examined the expression of genes involved in the uptake, transport, and synthesis of lipid content in the livers of mice fed diets containing cholesterol and quercetin. The expressions of carbohydrate-responsive elementbinding protein (also known as MLX interacting protein- 
A

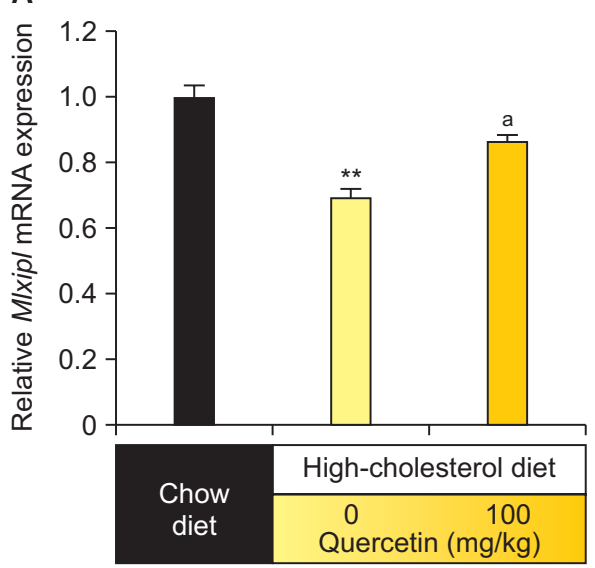

D

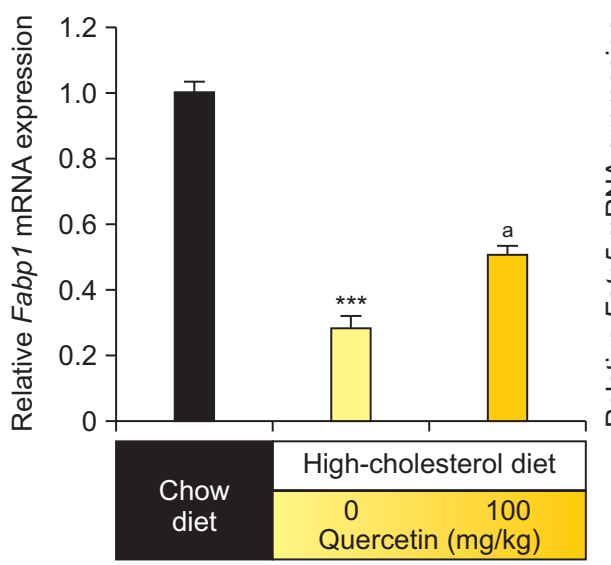

B

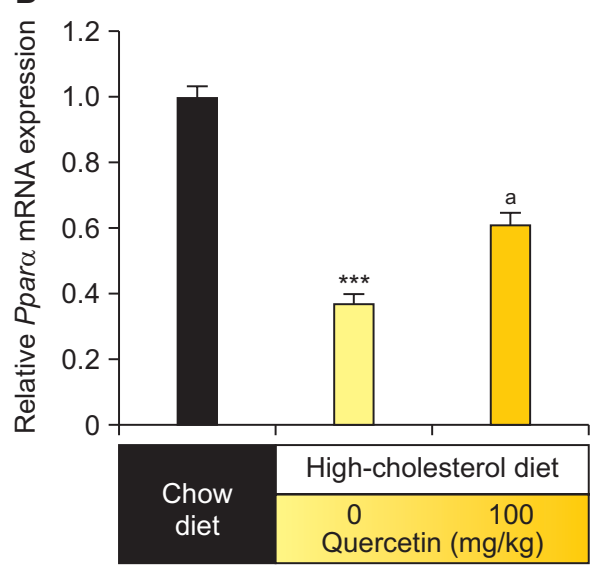

E

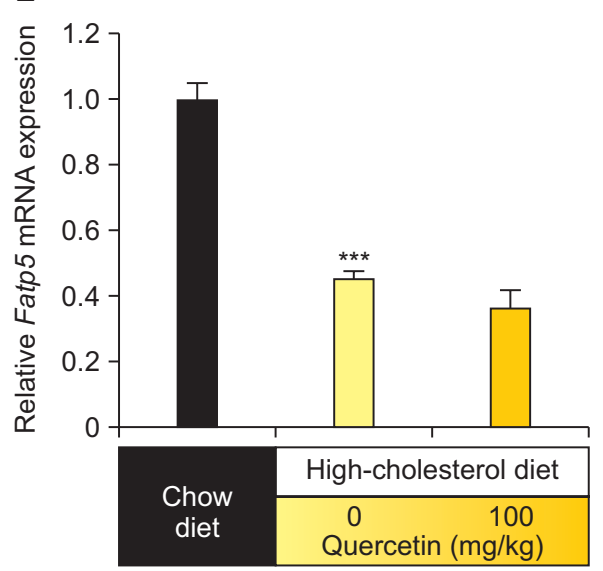

C

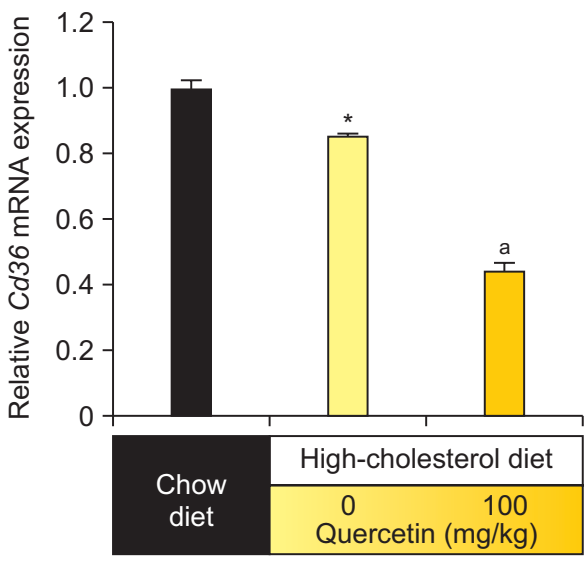

F

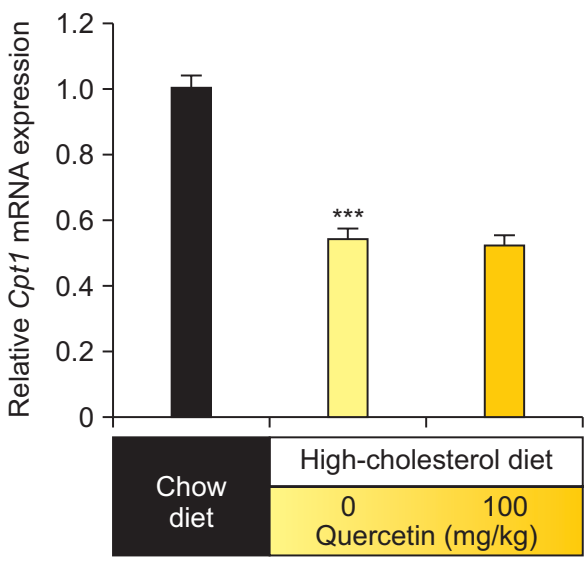

Fig. 6. Relative expression of genes involved in lipid metabolism in the liver of male mice fed a diet with high-cholesterol and quercetin $(100 \mathrm{mg} / \mathrm{kg})$. Quantitation of Mlxip/ (A), Ppara (B), Cd36 (C), Fabp1 (D), Fatp5 (E), and Cpt1 (F) was validated using quantitative RT-PCR. The asterisks denote significant differences $\left({ }^{*} p<0.05,{ }^{* *} p<0.01\right.$, and $\left.{ }^{* * *} p<0.001\right)$. The letter 'a' means that there is a significant difference in the expression of genes in the group with quercetin supplementation as compared to that in the highcholesterol diet group.

like (Mlxipl)), peroxisome proliferator-activated receptor alpha (Ppara), Cd36, fatty acid binding protein 1 (Fabp1), fatty acid transport protein 5 (Fatp5), and carnitine palmitoyltransferase I (Cpt1) were all significantly reduced by the high-cholesterol diet (Fig. 6). Among these genes, the expression of Mlxipl, Ppara, and Fabp1 was significantly recovered by quercetin supplementation. In contrast, the expression of $C d 36$ was further decreased in the liver by supplementation of quercetin, whereas the expression of Fatp5 and Cpt1 was not significantly affected by supplementation of quercetin.

\section{DISCUSSION}

In this study, we identified changes in the expression of Spam1, Adam5, Adam29, Sre5a2, and Sre5a3 involved in sperm maturation and sperm fertilizing ability in the testis tissues of mice fed normal chow and high-cholesterol diets. We also found that the addition of quercetin to a high-cholesterol diet mitigates changes in gene expression caused by cholesterol. In addition, a high-cholesterol diet with quercetin supplementation was shown to regulate the expression of genes related to lipid metabolism in the liver tissues of mice. These findings suggest that a disruption of cholesterol homeostasis poses a risk to normal sperm production and that quercetin has preventive effects on these abnormalities in male mice.

Several studies have reported that the expression of proteins involved in lipid metabolism can affect testicular function in mice (Wang et al., 2017; Sharma et al., 2019). Lipids and cholesterol are essential for Sertoli cells to remodel the membranes of germ cells. Therefore, cho- 
lesterol is needed to produce large amounts of germ cells during spermatogenesis. The absence of the 24-dehydrocholesterol reductase gene, the enzyme responsible for synthesizing cholesterol, causes infertility in male mice (Wechsler et al., 2003). Cholesterol is not only a major component of cell membranes but is also involved in intracellular signaling. In addition, cholesterol can be converted into bioactive substances such as steroid hormones, vitamin $\mathrm{D}$, and bile acids. The production of immature sperm is one of the main causes of male infertility. In a previous study, we identified changes in the expression of apolipoprotein D, a cholesterol-binding lipoprotein in male reproductive organs of rats fed a highcholesterol diet (Lim et al., 2016). However, no studies, to the best of our knowledge, have identified the expression of genes involved in sperm maturation and fertilization in the testes of mammals fed cholesterol.

SPAM1 is the most widely conserved mammalian sperm antigen and is expressed in both the testes and sperm of humans and rodents (Martin-Deleon, 2011). SPAM1 plays an important role in the penetration of sperm into the cumulus during fertilization. The ADAM family of proteins are distributed throughout the male reproductive track, including in the testes and epididymis (Edwards et al., 2008). Adam 1 and Adam 2 are involved in membrane interactions of the sperm and oocytes. Sperm in male mice deficient in Adam1 and Adam2 lack mobility in the oviduct and ability to bind to the membrane of oocytes (Cho et al., 1998; Kim et al., 2006a). Adam5 is located on the surface of mature sperm in mice (Kim et al., 2006b). Adam29 is also involved in sperm-oocyte interaction, and reduced expression of Adam29 is considered a potential indicator of male infertility (Schiza et al., 2019). Therefore, SPAM1, ADAM5, and ADAM29 are considered molecular markers of sperm maturation and sperm fertilizing ability (Jaiswal et al., 2015; Liu et al., 2019). SRD5A1 and SRD5A2 belong to the steroid $5 \alpha$-reductase family and are responsible for producing dihydrotestosterone, a derivative of testosterone (Saartok et al., 1984). In this study, a high-cholesterol diet reduced the expression of Spam1 and Adam29, whereas increased the expression of Adam5, Sre5a2, and Sre5a3 in the testes of mice. A high-cholesterol diet is suggested to affect male fertility by regulating the expression of genes involved in sperm maturation and sperm fertilizing ability. The association between these genes and the level of cholesterol in the body requires further study.

Quercetin has cytoprotective effects by regulating lipid metabolism in many types of cells. In glial cells, expression of sterol regulatory element-binding protein (Srebp)-1, Srebp-2, and Mlxipl at the mRNA and protein levels was reduced by quercetin (Damiano et al., 2019). Quercetin-3-glucoside, a glucoside form of quercetin, can alleviate hyperlipidemia and hyperinsulinemia in mice fed a high-cholesterol diet (Mbikay et al., 2018). Moreover, quercetin contributes to alleviating male reproductive toxicity caused by environmental factors. Quercetin restores a reduced number of Sertoli cells in mice treated with diesel exhaust particles (Izawa et al., 2008). Quercetin also modulates proteins in apoptotic pathways such as Bax, Bcl-xl, and caspase-3 in germ cells of male mice and mitigates the testicular toxicity of 4-nitrophenol (Mi et al., 2013). Quercetin also mitigates increased cholesterol levels and decreased $3 \beta$-hydroxysteroid dehydrogenase (HSD) and $17 \beta-H S D$ activity in the testes of rats exposed to cadmium (Ujah et al., 2018). High-cholesterol diets cause oxidative stress, inflammatory responses, and mitochondrial dysfunction in the pancreas of rats; however, a diet supplemented with quercetin mitigates these detrimental effects (Carrasco-Pozo et al., 2016). In addition, our study found that quercetin can mitigate changes caused by a high-cholesterol diet in gene expression in the testes of mice. Quercetin also has a protective effect against the negative effects of the herbicide atrazine on serum testosterone levels and the expression of CYP17A1, a major protein responsible for steroidogenesis in the testes of rats (Abdel Aziz et al., 2018). In humans and rodents, testosterone is synthesized mainly in Leydig cells, and maintaining proper concentrations of testosterone is essential for spermatogenesis (Woolveridge et al., 1998). This study also revealed that finasteride, which inhibits the conversion of testosterone to its most active form, regulates changes in genes involved in spermatogenesis, similar to the effects of quercetin.

The liver is the main organ that regulates the homeostasis of cholesterol in the body. The liver regulates absorption and synthesis to prevent cholesterol from accumulating in the plasma or tissues (Goldstein et al., 2006). Mlxipl is a glucose-sensing transcription factor that has been reported to contribute to hepatoprotection by preventing excessive cholesterol synthesis (Zhang et al., 2017). Ppara is responsible for lipid metabolism in the liver and 
its transcriptional regulation has been shown to prevent hepatotoxicity from cholesterol overload (Lu et al., 2019). CD36 is involved in the intracellular uptake of cholesterol and fatty acids (Nassir et al., 2007). FABP1 combines with various hydrophobic ligands, including fatty acids in the liver, to help prevent cytotoxicity. A high-cholesterol diet is known to inhibit the expression of FABP1 (Newberry et al., 2003; Martin et al., 2006). FATP5 is also involved in the transport of fatty acids in the liver, and the deletion of FATP5 has been shown to lower serum cholesterol levels (Doege et al., 2006). CPT1 is responsible for fatty acid transport to the mitochondria, regulating fatty acid oxidation (Irvin et al., 2014). In this study, we found that highcholesterol diets reduce the expression of lipid-related genes in the liver. The effects of quercetin in restoring the expression of genes that decreased under high-cholesterol conditions could be observed in Mlxipl, Ppara, and Fabp1. Further research is needed to determine whether quercetin can counterbalance the lipid metabolism in the liver caused by a high-cholesterol diet.

\section{CONCLUSION}

Taken together, we uncovered new genes related to sperm maturation and fertilization in male mice whose expression in the testes is altered with a high-cholesterol diet. In addition, confirmation that quercetin supplementation could reverse genetic changes caused by a highcholesterol diet suggests that quercetin is a potential biologically active substance that can prevent male infertility caused by a high-cholesterol diet.

\section{CONFLICTS OF INTEREST}

No potential conflict of interest relevant to this article was reported.

\section{ACKNOWLEDGEMENTS}

This research was supported by a grant of the National Research Foundation of Korea (NRF) grant funded by the Ministry of Science and ICT (MSIT) [grant number 2018R1C1B6009048]. We would also like to thank Institute of Animal Molecular Biotechnology in Korea University for supporting the publication fee.

\section{AUTHOR CONTRIBUTIONS}

C.Y., G.S.., and W.L. conceived and designed all experiments; C.Y., H.B. collected experimental samples and conducted all experiments; G.S., and W.L. analyzed, interpreted the data, and contributed to the development of the manuscript. All authors contributed to its critical review and agreed on the final version. All authors have read and agree to the published version of the manuscript.

\section{AUTHOR'S POSITION AND ORCID NO.}

\author{
C Yang, Ph.D. Candidate, \\ https://orcid.org/0000-0001-5326-9124 \\ H Bae, Ph.D., \\ https://orcid.org/0000-0002-9643-7797 \\ G Song, Professor, \\ http://orcid.org/0000-0003-2817-5323 \\ W Lim, Assistant Professor, \\ https://orcid.org/0000-0002-1328-0465
}

\section{REFERENCES}

Abdel Aziz RL, Abdel-Wahab A, Abo El-Ela FI, Hassan NEY, ElNahass ES, Ibrahim MA, Khalil AAY. 2018. Dose- dependent ameliorative effects of quercetin and l-Carnitine against atrazine- induced reproductive toxicity in adult male Albino rats. Biomed. Pharmacother. 102:855-864.

Brito AF, Ribeiro M, Abrantes AM, Pires AS, Teixo RJ, Tralhão JG, Botelho MF. 2015. Quercetin in cancer treatment, alone or in combination with conventional Therapeutics? Curr. Med. Chem. 22:3025-3039.

Carrasco-Pozo C, Tan KN, Reyes-Farias M, De La Jara N, Ngo ST, Garcia-Diaz DF, Llanos P, Cires MJ, Borges K. 2016. The deleterious effect of cholesterol and protection by quercetin on mitochondrial bioenergetics of pancreatic $\beta$-cells, glycemic control and inflammation: in vitro and in vivo studies. Redox Biol. 9:229-243.

Cavallini G. 2006. Male idiopathic oligoasthenoteratozoospermia. Asian J. Androl. 8:143-157.

Cho C, Bunch DO, Faure JE, Goulding EH, Eddy EM, Primakoff P, Myles DG. 1998. Fertilization defects in sperm from mice lacking fertilin beta. Science 281:1857-1859.

Damiano F, Giannotti L, Gnoni GV, Siculella L, Gnoni A. 2019. Quercetin inhibition of SREBPs and ChREBP expression results in reduced cholesterol and fatty acid synthesis in C6 glioma cells. Int. J. Biochem. Cell Biol. 117:105618.

Doege H, Baillie RA, Ortegon AM, Tsang B, Wu Q, Punreddy S, Hirsch D, Watson N, Gimeno RE, Stahl A. 2006. Targeted deletion of FATP5 reveals multiple functions in liver metab- 
olism: alterations in hepatic lipid homeostasis. Gastroenterology 130:1245-1258.

Edwards DR, Handsley MM, Pennington CJ. 2008. The ADAM metalloproteinases. Mol. Aspects Med. 29:258-289.

Goldstein JL, DeBose-Boyd RA, Brown MS. 2006. Protein sensors for membrane sterols. Cell 124:35-46.

Irvin MR, Aslibekyan S, Hidalgo B, Arnett D. 2014. CPT1A: the future of heart disease detection and personalized medicine? Clin. Lipidol. 9:9-12.

Izawa $\mathrm{H}$, Kohara $\mathrm{M}$, Aizawa $\mathrm{K}$, Suganuma $\mathrm{H}$, Inakuma $\mathrm{T}$, Watanabe G, Taya K, Sagai M. 2008. Alleviative effects of quercetin and onion on male reproductive toxicity induced by diesel exhaust particles. Biosci. Biotechnol. Biochem. 72:12351241.

Jaiswal MK, Agrawal V, Katara GK, Pamarthy S, Kulshrestha A, Chaouat G, Gilman-Sachs A, Beaman KD. 2015. Male fertility and apoptosis in normal spermatogenesis are regulated by vacuolar-ATPase isoform a2. J. Reprod. Immunol. 112:3845.

Kim E, Yamashita M, Nakanishi T, Park KE, Kimura M, Kashiwabara S, Baba T. 2006. Mouse sperm lacking ADAM1b/ ADAM2 fertilin can fuse with the egg plasma membrane and effect fertilization. J. Biol. Chem. 281:5634-5639.

Kim T, Oh J, Woo JM, Choi E, Im SH, Yoo YJ, Kim DH, Nishimura $\mathrm{H}$, Cho C. 2006. Expression and relationship of male reproductive ADAMs in mouse. Biol. Reprod. 74:744-750.

Leisegang K, Udodong A, Bouic PJ, Henkel RR. 2014. Effect of the metabolic syndrome on male reproductive function: a case-controlled pilot study. Andrologia 46:167-176.

Lim W, Bae H, Sohn JY, Jeong W, Kim SH, Song G. 2015. Dietary cholesterol affects expression of prostatic acid phosphatase in reproductive organs of male rats. Biochem. Biophys. Res. Commun. 456:421-427.

Lim W, Bae H, Song G. 2016. Differential expression of apolipoprotein $\mathrm{D}$ in male reproductive system of rats by high-fat diet. Andrology 4:1115-1122.

Liu Y, Liang C, Gao Y, Jiang S, He Y, Han Y, Olfati A, Manthari RK, Wang J, Zhang J. 2019. Fluoride interferes with the sperm fertilizing ability via downregulated SPAM1, ACR, and PRSS21 expression in rat epididymis. J. Agric. Food Chem. 67:5240-5249.

Lotti F, Corona G, Degli Innocenti S, Filimberti E, Scognamiglio V, Vignozzi L, Forti G, Maggi M. 2013. Seminal, ultrasound and psychobiological parameters correlate with metabolic syndrome in male members of infertile couples. Andrology 1:229-239.

Lu Y, Harada M, Kamijo Y, Nakajima T, Tanaka N, Sugiyama E, Kyogashima M, Gonzalez FJ, Aoyama T. 2019. Peroxisome proliferator-activated receptor $\alpha$ attenuates high-cholesterol diet-induced toxicity and pro-thrombotic effects in mice. Arch. Toxicol. 93:149-161.

Majumdar SS and Bhattacharya I. 2013. Genomic and postgenomic leads toward regulation of spermatogenesis. Prog. Biophys. Mol. Biol. 113:409-422.

Martin GG, Atshaves BP, McIntosh AL, Mackie JT, Kier AB,
Schroeder F. 2006. Liver fatty acid binding protein gene ablation potentiates hepatic cholesterol accumulation in cholesterol-fed female mice. Am. J. Physiol. Gastrointest. Liver Physiol. 290:G36-G48.

Martin-Deleon PA. 2011. Germ-cell hyaluronidases: their roles in sperm function. Int. J. Androl. 34(5 Pt 2):e306-e318.

Mbikay M, Mayne J, Sirois F, Fedoryak O, Raymond A, Noad J, Chrétien M. 2018. Mice fed a high-cholesterol diet supplemented with quercetin-3-glucoside show attenuated hyperlipidemia and hyperinsulinemia associated with differential regulation of PCSK9 and LDLR in their liver and pancreas. Mol. Nutr. Food Res. 62:e1700729.

Mi Y, Tu L, Wang H, Zeng W, Zhang C. 2013. Supplementation with quercetin attenuates 4-nitrophenol-induced testicular toxicity in adult male mice. Anat. Rec. (Hoboken) 296:16501657.

Nassir F, Wilson B, Han X, Gross RW, Abumrad NA. 2007. CD36 is important for fatty acid and cholesterol uptake by the proximal but not distal intestine. J. Biol. Chem. 282:1949319501.

Newberry EP, Xie Y, Kennedy S, Han X, Buhman KK, Luo J, Gross RW, Davidson NO. 2003. Decreased hepatic triglyceride accumulation and altered fatty acid uptake in mice with deletion of the liver fatty acid-binding protein gene. J. Biol. Chem. 278:51664-51672.

Saartok T, Dahlberg E, Gustafsson JA. 1984. Relative binding affinity of anabolic-androgenic steroids: comparison of the binding to the androgen receptors in skeletal muscle and in prostate, as well as to sex hormone-binding globulin. Endocrinology 114:2100-2106.

Saez Lancellotti TE, Boarelli PV, Monclus MA, Cabrillana ME, Clementi MA, Espínola LS, Cid Barría JL, Vincenti AE, Santi AG, Fornés MW. 2010. Hypercholesterolemia impaired sperm functionality in rabbits. PLoS One 5:e13457.

Samova S, Patel CN, Doctor H, Pandya HA, Verma RJ. 2018. The effect of bisphenol A on testicular steroidogenesis and its amelioration by quercetin: an in vivo and in silico approach. Toxicol. Res. (Camb) 7:22-31.

Schiza C, Korbakis D, Jarvi K, Diamandis EP, Drabovich AP. 2019. Identification of TEX101-associated proteins through proteomic measurement of human spermatozoa homozygous for the missense variant rs35033974. Mol. Cell. Proteomics 18:338-351.

Sharma S, Ahmad S, Afjal MA, Habib H, Parvez S, Raisuddin S. 2019. Dichotomy of bisphenol A-induced expression of peroxisome proliferator-activated receptors in hepatic and testicular tissues in mice. Chemosphere 236:124264.

Ujah GA, Nna VU, Agah MI, Omue LO, Leku CB, Osim EE. 2018. Effect of quercetin on cadmium chloride-induced impairments in sexual behaviour and steroidogenesis in male Wistar rats. Andrologia 50.

Wang F, Chen Z, Ren X, Tian Y, Wang F, Liu C, Jin P, Li Z, Zhang F, Zhu B. 2017. Hormone-sensitive lipase deficiency alters gene expression and cholesterol content of mouse testis. Reproduction 153:175-185. 
Wang HJ, Wang Q, Lv ZM, Wang CL, Li CP, Rong YL. 2015. Resveratrol appears to protect against oxidative stress and steroidogenesis collapse in mice fed high-calorie and highcholesterol diet. Andrologia 47:59-65.

Wechsler A, Brafman A, Shafir M, Heverin M, Gottlieb H, Damari G, Gozlan-Kelner S, Spivak I, Moshkin O, Fridman E, Becker Y, Skaliter R, Einat P, Faerman A, Björkhem I, Feinstein E. 2003. Generation of viable cholesterol-free mice. Science 302:2087.

Woolveridge I, Bryden AA, Taylor MF, George NJ, Wu FC, Morris ID. 1998. Apoptosis and expression of apoptotic regula- tors in the human testis following short- and long-term antiandrogen treatment. Mol. Hum. Reprod. 4:701-707.

Yokoyama S. 2000. Release of cellular cholesterol: molecular mechanism for cholesterol homeostasis in cells and in the body. Biochim. Biophys. Acta 1529:231-244.

Zhang D, Tong X, VanDommelen K, Gupta N, Stamper K, Brady GF, Meng Z, Lin J, Rui L, Omary MB, Yin L. 2017. Lipogenic transcription factor ChREBP mediates fructose-induced metabolic adaptations to prevent hepatotoxicity. J. Clin. Invest. 127:2855-2867. 